\title{
Surface roughness as rupture control factor of lipid vesicles
}

\author{
A.A. Duarte* and M. Raposo* \\ *CEFITEC, Departamento de Física, Faculdade de Ciências e Tecnologia, FCT, Universidade \\ Nova de Lisboa, 2829-516 Caparica, Portugal
}

Liposomes or lipid vesicles are self-closed structures formed by one or several concentric lipid bilayers with an aqueous phase inside, which may incorporate almost any molecule, namely proteins, hormones, enzymes, antibiotics, anticancer agents, antifungical agents, gene transfer agents, DNA, and whole viruses. Scientific evidences prove that unprotected liposomes containing drugs are easily released from the endoplasmic reticulum of the cell [1]. To increase the vesicles lifetime and to activate a controlled drug release with an external stimulus, the vesicles immobilization on a surface and the factors which create conditions to the liposome rupture have to be analyzed. A number of studies have identified some of the critical stages of vesicle adsorption (adhesion), fusion, deformation, rupture, and spreading of the lipid bilayer. Nevertheless, the formation mechanisms of well-controlled continuous supported bilayers or adsorption of whole liposomes are still not fully understood. As yet it was demonstrated that a controlled adsorption of vesicles containing a small fraction of charged lipids occurs without rupture and their subsequent embedding in polyelectrolyte multilayer (PEM) films, meaning vesicles may be immobilized in an intact or slightly deformed state, which can act as drug reservoirs [2-5]. Moreover, depending on the nature of the physicochemical conditions of the vesicle solution and the substrate surface, a flat lipid bilayer can be formed, known as supported lipid bilayers, which can incorporate membrane proteins and keep the native dynamics of the lipid bilayer mimicking a biological membrane [6-10]. In this study, a layer of 1,2-dipalmitoyl-sn-glycero-3-[phospho-rac-(1-glycerol)] (sodium salt) (DPPG) liposomes adsorbed onto PEMs cushions based on poly(ethylenimine) (PEI), poly(sodium 4styrenesulfonate) (PSS) and poly(allylamine hydrochloride) (PAH) polyelectrolytes was analyzed by atomic force microscopy (AFM) technique in non-contact mode and quartz crystal microbalance (QCM).

Sequential heterostructures of Si/PEI(PSS/PAH) ${ }_{4}$ and $\mathrm{Si} / \mathrm{PAH}$, also designated cushions, were prepared onto silicon substrates using the layer-by-layer $(\mathrm{LbL})$ technique [11] with polyelectrolyte solutions of PEI, PSS and PAH of monomeric concentrations of $0.01 \mathrm{M}$. Topographic images of $1 \times 1 \mu \mathrm{m}^{2}$ area of Si/PAH/DPPG (Figure $1 \mathrm{a}$ ), and Si/PEI(PSS/PAH) 4 /DPPG (Figure 1 b) LbL films were acquired by AFM. The root mean square roughness (RMS) calculated from topographies data are listed in table I. As shown, when a DPPG layer is adsorbed onto Si/PAH the RMS keeps an approximately equal value meaning that the liposome disrupted and spread onto the surface forming a planar lipid bilayer. But when a DPPG layer is adsorbed onto $\mathrm{Si} / \mathrm{PEI}(\mathrm{PSS} / \mathrm{PAH})_{4}$ the RMS value doubled, indicating that the structural integrity of the liposomes is maintained, even though there has been any deformation during adsorption. The adsorbed amount of the two PEMs and DPPG-liposomes layers was measured using a QCM and is displayed in table I. The DPPG adsorbed amount obtained on the PAH cushion was approximately equal to a planar lipid bilayer [12], while the adsorption onto PEI(PSS/PAH) ${ }_{4}$ was higher than the predicted for a planar lipid bilayer. This behavior suggests that the DPPG liposomes on the second PEM remained intact during adsorption. Both confirm the AFM results. Therefore we conclude that the initial roughness of the surface is a primordial factor to determine the adsorption or not of intact vesicles.

\section{References}

1. Liu A.L., Advances in planar lipid bilayers and liposomes, volume 8, USA: Academic Press, 2008.

2. Min Y. et al., Langmuir, 26:8684-8689, 2010.

3. Gromelski S. et al., Colloids Surf. B Biointerfaces, 74:477-483, 2009.

4. Vu T.H. et al., J.. Colloid Interface Sci., 336:902-907, 2009. 
5. Richter R. et al., Biophys J., 85:3035-3047, 2003.

6. Keller C.A. et al., Phys. Rev. Lett. 84:5443-5446, 2000.

7. Reimhult E. et al., Int. J. Mol. Sci., 10:1683-1696, 2009.

8. Reimhult E. et al., Langmuir, 19:1681-1691, 2003.

9. Anderson T.H. et al., Langmuir, 25:6997-7005, 2009.

10. Weirich K.L. et al., Biophys. J., 98:85-92, 2010.

11.Decher G. et al., Thin Solid Films, 210:831-835, 1992.

12.Duarte, A.A. et al., submitted

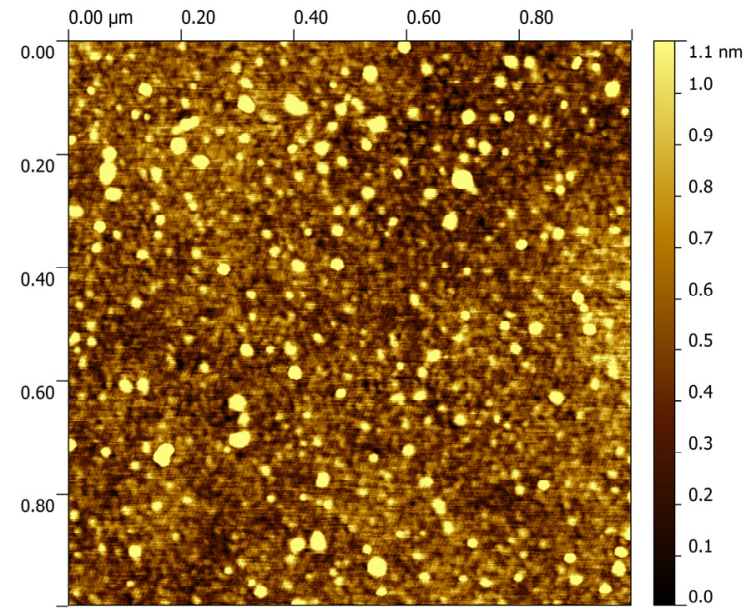

a)

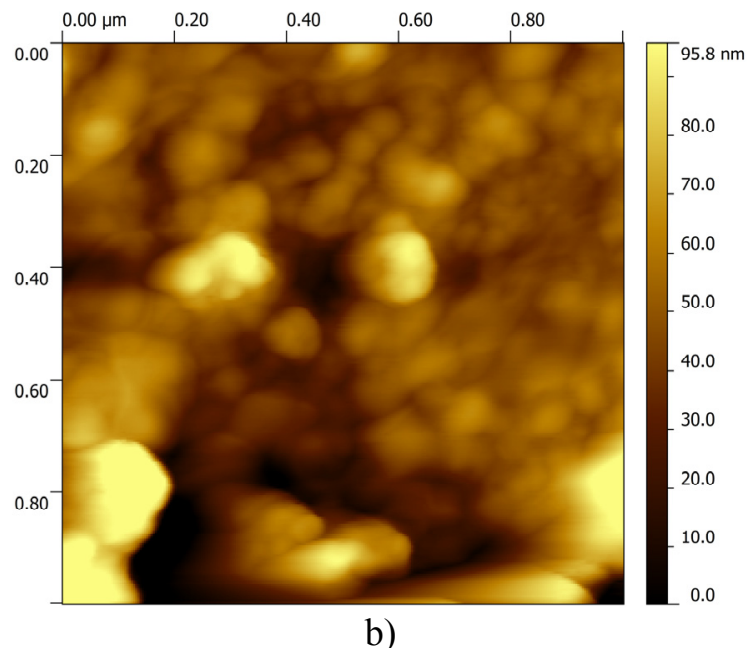

b)

Figure 1. AFM topographic images with $1 \mathrm{x} 1 \mu \mathrm{m}^{2}$ area of a) Si/PAH/DPPG and b) $\mathrm{Si} / \mathrm{PEI}(\mathrm{PSS} / \mathrm{PAH})_{4} / \mathrm{DPPG}$ LbL films.

Table I. RMS values obtained by statistical treatment of the AFM images, experimental values of the adsorbed amount obtained by QCM and High-resolution vacuum ultraviolet (VUV) [12] of the PAH, PAH/DPPG, PEI(PSS/PAH) $)_{4}$ and PEI(PSS/PAH) $)_{4} / \mathrm{DPPG}$ LbL films.

\begin{tabular}{|l|c|c|}
\hline LbLFilm & $\begin{array}{c}\mathbf{R}_{\text {MS }} \\
(\mathbf{n m})\end{array}$ & $\begin{array}{c}\text { Adsorbedamount } \\
\left(\mathbf{m g} / \mathbf{m}^{\mathbf{2}} \mathbf{)}\right.\end{array}$ \\
\hline $\mathrm{PAH}$ & 0.2 & $1.4 ; 0.34^{*}[12]$ \\
\hline $\mathrm{PAH} / \mathrm{DPPG}$ & 0.3 & $6.7 ; 5.34^{*}[12]$ \\
\hline $\mathrm{PEI}(\mathrm{PSS} / \mathrm{PAH})_{4}$ & 10.9 & 93 \\
\hline $\mathrm{PEI}(\mathrm{PSS} / \mathrm{PAH})_{4} / \mathrm{DPPG}$ & 20.3 & 137 \\
\hline
\end{tabular}

The authors acknowledge the "Fundação para a Ciência e Tecnologia" (FCT-MEC) by the postgraduate scholarship SFRH/BD/62229/2009 and the "Plurianual" funding. 